\title{
Higgs Field and Gauge Invariance in Spacetime Transformations
}

\author{
Yougang Feng \\ College of Physics, Guizhou University, Guiyang, China \\ Email: ygfeng45@aliyun.com
}

How to cite this paper: Feng, Y.G. (2017) Higgs Field and Gauge Invariance in Spacetime Transformations. Journal of Modern Physics, 8, 448-458.

https://doi.org/10.4236/jmp.2017.84029

Received: February 7, 2017

Accepted: March 7, 2017

Published: March 10, 2017

Copyright $\odot 2017$ by author and Scientific Research Publishing Inc. This work is licensed under the Creative Commons Attribution International License (CC BY 4.0).

http://creativecommons.org/licenses/by/4.0/

\begin{abstract}
The concepts of the mass charge and the Higgs potential are proposed. The generation of the Higgs potential is a mutation, and the particles' masses originate from the mechanism that the particles with mass charges obtain static energies under the potential, and the energies are regarded as the potential energies of the particles in the Higgs field, shared jointly by the field and the particles. The mass charge as an intrinsic property of a particle cannot change with its motion, and the potential's change obeys the Lorentz transformation. When time warps the massless particles get masses, which are tensors. There is gauge invariance in the self-similar transformations of the spacetime, resulting in superlight and the cosmic inflation with the maximum speed more than five times light velocity, and the universe has been so far expanding at the superlight speed due to the dark energies (two thirds of the universe energies). An explicit figure for the creation of four types of Higgs fields is drawn. The orders of magnitudes of three characteristic temperatures relating to the time phase transitions are estimated. Finally, the effect of the Higgs field on the weak interaction and the spins' chirality is discussed.
\end{abstract}

\section{Keywords}

Mass, Higgs, Potential, Gauge, Superlight, Inflation

\section{Introduction}

One of the most efficient methods in physics is the consideration of the symmetry of a physical system. Group theory and representation theory are the powerful tools for handling the symmetries of such a system. However, many patterns of nature are very complicated and irregular, in front of which the traditional geometry looks weak [1]. The degree of their irregularity and fragmentation is usually identical in all scales, which means there are fractal structures. Meanwhile those acquainted symmetry theories used in the conventional quantum 
theory cannot be directly applied in the analysis on fractals. Self similarities and hierarchies are required to be firstly considered. The spacetime lattice model revealed that in the evolution of the universe there had been fractal structures for the spacetime with higher dimensions than four [2] [3]. The model shows us since the big bang generated the universe temperature is bound to have a decisive effect upon its growth process. The spontaneous breaking of the time local symmetry is an irreversible process and can be described by the renormalization group theory. In addition, according to the space lattice model, the vibration of the space lattices represents the fluctuation of thermal radiation energy, and the $\mathrm{S}$ bosons as the quanta of the radiation fluctuation should be matter particles, different from the quasiparticles such as the phonons, the quanta of the crystal lattice vibration. The time model suggests that the time field plays a main character in the standard model without which there could be no local symmetry-breaking. The time field is just the Higgs field.

The standard model has uniformly solved the Higgs mechanism and the Nambu-Goldstone mechanism by two ways on the imaginary plane [4]: one is on the real axis, and another is on the imaginary axis. However, the imaginary way doesn't exist in our real world. We think the combination of the dynamic lattice model and the static model in the spacetime model is a better substitution for the ways [3]: the particles get masses through the Higgs mechanism in the static lattice model; the particles in the dynamic lattice model are still massless at the same time. The fact that the Higgs particles obtain masses in the Higgs field implies that the interaction of the Higgs particles and others may not the mechanism for getting other particles' masses. Moreover, unlike the electric charge, the particle's mass may not be the intrinsic property. Otherwise, it wouldn't change in the relative motion. It seems that we should find more reasonable account for the mechanism. It is necessary to give an explicit physical pattern for the fractal structures of spacetime in order to study the connection between the normal spacetime and the fractal spacetime, especially the gauge invariance in the transformations. Talking about the transformation depends on the specific temperatures linking to the Higgs field. To ignore the temperature makes it impossible for us to understand the details of the critical behavior and the specific process for the spontaneous breaking of the local time symmetry, hindering our learning of the nature of the Higgs field and the real cause for the getting particle's mass. Thus, the estimation of some characteristic temperatures should be done. The nature that the Higgs field is the time field contributes to our understanding about what role the field plays in the weak interaction. Up to this point, we set out to prove it.

In Section 2, introducing the mass charge and the Higgs potential, we explain the mechanism for the getting of mass and give an explicit figure for the selfsimilar transformations, in which gauge invariance is found, leading to superlight in the fractal structures. In Section 3, we discuss four types of Higgs fields and three characteristic temperatures for the time phase transitions, and their orders of magnitudes are estimated. We find the temperature region of the cos- 
mic inflation, and the maximum inflation speed is calculated. We conclude that the universe has been so far expanding due to the dark energies, which are two thirds of the universe energies (about 66.7\%). Finally, we discuss how the Higgs field effects statistically on the weak interaction and the spins' chirality. Section 4 is conclusion remark.

\section{Theory}

\subsection{Mass Charge and Higgs Potential}

The so-called independent freedom degree in the principle of the partition of the independent freedom degrees refers to the independent dimension [3], the real time is an independent one. Let $Q_{m}$ be a mass charge for a particle, $V_{H}$ be the potential function of the Higgs field, the particle obtains its static energy $E_{0}$ under the potential

$$
E_{0}=Q_{m} V_{H}
$$

$V_{H}$ is a physical parameter on the time dimension; it has vanishing components in the space as the orthogonality between them, and

$$
V_{H}=\left(V_{H 0}, V_{H 1}, V_{H 2}, V_{H 3}\right), V_{H 1}=V_{H 2}=V_{H 3}=0
$$

where $V_{H \mu}, \mu=0,1,2,3$, is the component in the time and space, respectively. It should be emphasized that the static energy is the potential energy of the particle in the Higgs field, shared jointly by the field and the particle. The mass is given by

$$
m=E_{0} / c^{2}=Q_{m} V_{H} / c^{2}
$$

where $c$ is the light speed. As a quantum parameter, $V_{H}$ is proportional to the time-order intensity at absolute zero $M(0)$ [2], and

$$
V_{H}=\eta M(0)
$$

where $\eta$ is a constant. Since the Higgs particles condense, the $M(0)$ has a saturated value, hence $V_{H}$ is a constant.

We can construct a field (the space field) variable $A(x)$ by means of the property of the S bosons [3], and combining Equation (1), we get the KleinGorden field equation

$$
\left(\partial^{\mu} \partial_{\mu}+Q_{m}^{2} V_{H}^{2}\right) A(x)=0
$$

We take $c=\hbar=1$ unit. Since $\partial^{\mu} \partial_{\mu} A(x)=0$, so $Q_{m} V_{H}=0$. The mass charge is the intrinsic property of the $\mathrm{S}$ boson, it cannot change, thus $Q_{m} \neq 0$. Therefore,

$$
V_{H}=0
$$

Because the $A(x)$ is independent of time [3], i.e., the $\mathrm{S}$ bosons move at the light speed in the space, except the time dimension. So the $V_{H}$ in Equation (5) should be the potential's components in the space, $V_{H i}, i=1,2,3$, rather than the $V_{H 0}$.This consequence accords with Equation (2), vice versa. Equation (6) opens to us that if a particle is independent of the time dimension, available to 
the dynamic lattice model, the relevant $V_{H}=0$; conversely, fitting to the static lattice model and the relevant $V_{H} \neq 0$, being constant.

In the relative motion, $V_{H}$ changes on the Lorentz transformation,

$$
V_{H}^{\prime}=V_{H} / \sqrt{1-(u / c)^{2}}
$$

It results in the change of the mass for a particle moving along the $\mathrm{x}\left(\mathrm{x}^{\prime}\right)$ axis at the velocity $u$ with respect to the $V_{H}$, and the mass is

$$
m^{\prime}=\frac{Q_{m}}{c^{2}} \cdot \frac{V_{H}}{\sqrt{1-(u / c)^{2}}}
$$

The mass charge is the proper physical property of the particle, not changing with the motion. For the particle moving at the light speed, Equation (7) makes the potential no sense, since the particle is independent of the time in the dynamic lattice model [3]. The relevant energy-momentum relation becomes

$$
E^{2}=c^{2} p^{2}+Q_{m}^{2} V_{H}^{2}
$$

where $E$ and $p$ are respectively the energy and the momentum for the particle. We may let the unit of the mass charge be $Q_{m}=m$ in the quantum theory, then the potential will be $V_{H}=1$ if the unit of $c=\hbar=1$ is taken.

Except quarks and those composite particles like the protons and neutrons containing internal structures, all of elementary matter particles have their own mass charges, even if for those massless particles such as $\mathrm{S}$ bosons, photons, and gravitons. Both a particle and its antiparticle have the same mass charge, and the charges of particles of different types are distinguishable from each other. Since the particles sharing the time dimension only have limited moving speed, and they always immerge in the Higgs potential, which is full of the whole time dimension, such that there is no negative mass. The uniqueness of the mass charge leads to that unlike the electric neutrality for the electric charges, there is no the mass neutrality for the mass charges. The mass conservation depends only on the static energy conservation, if the mass conservation holds, so does the mass charges.

Let's set up a man-made reference coordinate system, its time axis is always orthogonal to the space, no matter whether or not warps the real tome, i.e., the natural time. The field of the natural time does be the Higgs field. When the natural time warps, the Higgs potential varies with the space position, it may be represented by a $4 \times 4$ matrix in the reference system as the following

$$
V_{H}=\left[\begin{array}{cccc}
V_{H 00} & V_{H 01} & V_{H 02} & V_{H 03} \\
V_{H 10} & V_{H 11} & V_{H 12} & V_{H 13} \\
V_{H 20} & V_{H 21} & V_{H 22} & V_{H 23} \\
V_{H 30} & V_{H 31} & V_{H 32} & V_{H 33}
\end{array}\right]
$$

The particle gets its static energy $E_{H}$ under the potential $V_{H}$ :

$$
E_{H}=Q_{m} V_{H}, E_{H \lambda \mu}=Q_{m} V_{H \lambda \mu}, \lambda, \mu=0,1,2,3
$$

where the $E_{H \lambda \mu}$ is the components of the $E_{H}$. The $S$ bosons, photons, and gravitons will become massive particles, their masses behave in a tensor form 


$$
m=\frac{E_{H}}{c^{2}}, m_{\lambda \mu}=E_{H \lambda \mu} / c^{2}
$$

where the $m_{\lambda \mu}$ is the components of the $m$.

\subsection{Gauge Invariance and Superlight}

\subsubsection{Two Descriptions for the Transformations}

An ordered block of the cubic lattice model exists really in the 3-dimensional space, however, it's impossible to compute accurately the block's ordered state in the space. Reference [5] tells us that it can be solved in a higher dimensional space by means of topological analysis: the block is equivalent to a $D^{4}$ -dimensional simple connected space, where the $D$ is the fractal dimension of its sub-block. An explicit explanation of the self-similar transformations conduces to understand the reason why there exists the gauge invariance. There are two descriptions for the transformations: direct and equivalent. The spacetime lattice model is topologically the cubic lattice system, its transformations can be directly described by the self-similar transformation theory [5], which is called the direct description: On the zero-th hierarchy there are only infinite original lattices, some of them form a first-order sub-block of $D$-dimension on the first hierarchy, and the original lattices become the lattices in the inner space of the first-order sub-block. On the first hierarchy some the first-order sub-blocks make up a first-order block with $D^{4}$-dimension, these sub-blocks map into the lattices in the first-order block at the same time. Thus, the first time self-similar transformation succeeds on the first hierarchy, and the blocks have the same symmetry as the original lattices'. On the $r$-th hierarchy, $1<r<+\infty$, there are $r$-order sub-blocks, each sub-block contains lattices, each of which is the $(r-1)$-order block on the $(r-1)$-th hierarchy. On this $r$-th hierarchy some $r$-order sub-blocks construct a $r$-order block, and these $r$-order sub-blocks become the lattices in the $r$-order block. Only if $r \rightarrow+\infty$, the whole system is an ordered block, and the system turns into the ordered state, otherwise disordered.

Equivalent description: Because there is no interaction between the sub-block and the block [5], from the point of view of the interaction the system is viewed as a reducible system including two independent irreducible subsystems: the sub-blocks subsystem and the blocks subsystem. The two subsystems exist on the same hierarchy. Each subsystem has its own self-similar transformation: 1 . Some $(r-1)$-order sub-blocks of the $(r-1)$-th hierarchy form a $\mathrm{r}$-order sub-block on the $\mathrm{r}$-th hierarchy, and these $(r-1)$-order sub-blocks become the lattices in the r-order sub-block. 2. Some $(r-1)$-order blocks of the $(r-1)$-th hierarchy produce cooperatively a r-order block on the $\mathrm{r}$-th hierarchy, these $(r-1)$-order blocks shrinks into the lattices in the r-order block.

\subsubsection{Gauge Invariance}

It's necessary to reaffirm the scaling law here, although it has been described in the reference [5]. The rule of the hierarchy is that the $r$-order sub-blocks (blocks) exist only on the $r$-th hierarchy, they should shrink into the lattices on the $(r+1)$-th hierarchy; as a lattice the sub-block (block) has no internal structure. 
The scaling law concerns with the distinguishability of the internal space of a sub-block (block), relating to two cases: 1 . If we describe the system by using the interaction between the $r$-order sub-blocks (blocks) and omit the interaction between the lattices in the sub-blocks (blocks) as the illustration of the Formula (7) in the reference [5], the internal space of each sub-block (block) should be thought of as indistinguishable on the $r$-th hierarchy although the sub-blocks (blocks) are not the lattices, and their outside space is just the 4-dimensional spacetime. 2. If we consider only the similar structures of the $r$-order sub-blocks (blocks), the internal space of each sub-block (block) is distinguishable with a definite fractal dimension. For the first case, there are infinite sub-blocks on the $r$-th hierarchy, $1<r<+\infty$, we make the symmetric center of a $r$-order sub-block represent it, the radius of the sphere covering the center equals $R_{s}=c t_{s}$, where $c$ is the light speed, and $t_{S}$ is the time element of the sub-block, the orientation of the $t_{S}$ can be either positive or negative. The sphere equation is

$$
\Delta x_{1}^{2}+\Delta x_{2}^{2}+\Delta x_{3}^{2}=c^{2} t_{S}^{2}
$$

The coordinates of a point on the sphere is $x_{i}, i=1,2,3$, the left side of Equation (13) is the square of the distance between the sphere center and the point. Since the outside space of the sub-block is the 4-dimensional spacetime, Equation (13) manifests the Lorentz gauge. In the second case mentioned above, the r-order sub-block is just the lattice in a $(r+1)$-order sub-block on the $(r+1)$-th hierarchy, the radius of the sphere covering this lattice is $R_{S L}=v_{S} \tau$, where $v_{S}$ is the velocity at which the massless particles move in the inner space of the sub-block, $\tau$ is the lattice's time element and its orientation takes positive or negative. Like the Equation (13), its sphere equation is expressed as

$$
\Delta x_{1}^{2}+\Delta x_{2}^{2}+\cdots+\Delta x_{D}^{2}=v_{S}^{2} \tau^{2}
$$

The outside spacetime of the lattice does be the inner spacetime of the subblock, which is $(D+1)$-dimensions (the space is $D$-dimensional). The r-order sub-block and the lattice in the $(r+1)$-order sub-block are the same object. As the self-similar transformation the scale should be invariant, thus

$$
c^{2} t_{S}^{2}=v_{S}^{2} \tau^{2}
$$

With the same reason, for the $r$-order block on the $r$-th hierarchy its covering sphere leads to equation

$$
\Delta x_{1}^{2}+\Delta x_{2}^{2}+\Delta x_{3}^{2}=c^{2} t_{B}^{2}
$$

where $t_{B}$ is the time element of the block, it can be positive or negative. The same object becomes the lattice in the $(r+1)$-order block, the equation for its covering sphere follows

$$
\Delta x_{1}^{2}+\Delta x_{2}^{2} \cdots+\Delta x_{D^{4}}^{2}=v_{B}^{2} \tau^{2}
$$

The outside spacetime of the lattice is the inner spacetime of the block (the space is $D^{4}$-dimensions), $v_{B}$ is the velocity at which the massless particles move in the $\left(D^{4}+1\right)$-dimensional spacetime. As the scaling law, all of lattices 
have the same time element $\tau$, just like the lattice spin $s$ in the Ising model [5]. By the scaling law, Equations (16) and (17) yield

$$
c^{2} t_{B}^{2}=v_{B}^{2} \tau^{2}
$$

Equations (15) and (18) imply gauge invariance in the transformations. The gauge belongs only to the metric space, which here is the spacetime, and as the scale the length standard at each spacetime point is of locality. The so-called scale is also named after the gauge. The self-similar transformations keep the gauge constant in local range. All of these fractal structures with invariant gauge make up of a gauge system. It is just the spacetime itself. In this sense, the properties of the gauge fields exhibit the intrinsic properties of the spacetime.

\subsubsection{Superlight}

The one-to-one relation between the time lattice model and the Ising model suggests that the sub-block's time element $t_{S}$ corresponds to the sub-block's spin $S_{S}$, the block's time element $t_{B}$ to the block's spin $S_{B}$, and the lattice's time element $\tau$ to the lattice's spin $s$. Let $\tau=1$, like $s=1$ [5]. Using the Formula (6.1) of the reference [5], we have

$$
t_{S}=\sqrt{2 D / Z_{1}}
$$

where $Z_{1}=4$ is the coordinate number for the sub-block of the cubic lattice, and $D$ is its fractal dimension, hence

$$
t_{S}=\sqrt{2 D} / 2
$$

Similarly, the block's time element $t_{B}$ is given by the Formula (6.2) for the same reference, and

$$
t_{B}=\sqrt{2 D^{k} / Z_{2}}
$$

where $Z_{2}=6, k=4$ are the block's coordinate number and the number of sub-blocks in a block. Thus,

$$
t_{B}=(\sqrt{3} / 3) D^{2}
$$

Combining Equations (15) and (18), we get

$$
v_{S}=t_{S} c=(\sqrt{2 D} / 2) c, v_{B}=t_{B} c=(\sqrt{3} / 3) D^{2} c
$$

Obviously, $v_{S}>c, v_{B}>c$, since $D>2$ (see the Formula (5) of the same reference). The consequence signifies that there are superlight phenomena in the fractal structures. Since the fractal dimension $D$ is constant at a fixed temperature, the similarity between $v_{S}$ and $c$, or $v_{B}$ and $c$ (the linear relationship between them) indicates the superlight is a necessary condition for the maintaining of the gauge in different dimensional spacetime. The $c, v_{S}$, and $v_{B}$ are actually the constant quantities for the same gauge, their values depend on the dimensions of the relevant spacetime.

\section{Discussion}

\subsection{Four Types of Higgs Fields}

Consider the direct description said in the subsection 2.2.1. On the first hie- 
rarchy the original lattices form a sub-block in an ordered time state, these lattices make up the first type of Higgs field. Infinite sub-blocks construct the second type of Higgs field, and cooperate to retain the field in the disordered time state. The lattices in a block correlate to each other to build up the third type of Higgs field, and the block is in an ordered time state. The fourth type of the field is set up by infinite blocks, and the time is in disordered state for the field. After infinitely hierarchical transformations the system's time is ordered, meanwhile the temperature is in the region of $T_{E W}<T \leq T_{C}$. The three types of Higgs fields disappear at $T_{E W}$, except the first type, which becomes the Higgs field to give the particles masses. The Higgs field is a mutant, too, the occurrence of Higgs particles. That the Higgs particles have masses is an evidence for the field's self-energy.

\subsection{Three Characteristic Temperatures}

Weinberg pointed out that the radiation would have continued to predominate over matter until the temperature dropped to $400{ }^{\circ} \mathrm{K}$ [6]. If his mention is true, the estimated critical temperature $T_{B E}$ of the Bose-Einstein condensation phase transition of the time may be in the order of magnitude of about $4 \times 10^{2}{ }^{\circ} \mathrm{K}$. Making use of the gauge theories, D.A. Kirzhnits and A.D. Lind got the transition temperature $T_{E W}$ of about $3 \times 10^{15}{ }^{\circ} \mathrm{K}$ for the electro-weak phase transition [7]. In the following we try to estimate the critical temperature $T_{C}$ for the time order-disorder phase transition. According to the reference [5], the equivalent description get the critical point $K_{C}$ of the system, which is the sum of the critical point $K_{C 1}$ of the sub-blocks subsystem and the critical point $K_{C 2}$ of the blocks subsystem:

$$
K_{C}=K_{C 1}+K_{C 2}
$$

and

$$
K_{C 1}=J_{1} /\left(k_{B} T_{C}\right)=1 /\left(2 D_{\min }\right), K_{C 2}=J_{2} /\left(k_{B} T_{C}\right)=1 /\left(2 D_{\text {min }}^{4}\right)
$$

where $J_{1}$ and $J_{2}$ are the coupling constants for the sub-blocks and the blocks, respectively, $k_{B}=0.00008617 \mathrm{eV} /{ }^{\circ} \mathrm{K}$ is Boltzmann constant, $D_{\min }=2.4781$ is the minimum fractal dimension for the cubic [5]. The $D_{\min }$ corresponds to a fractal side $n^{*}=4.7891$, which doesn't accords with the transformation since only the integer sides can keep the original symmetry. We use the mean value $\langle D\rangle=2.4807$ of the fractal dimensions relating to the integer sides $n_{1}=4$ and $n_{2}=5$ instead of the $D_{\min }$. We notice that rather than the Higgs field, the strong interaction is mainly responsible for the quarks' static energies; on the other hand the coupling constant is the exchange integral associated with the interaction. The critical temperature $T_{C}$ should guarantee the creation of the quarks, this requires that the constant $J_{2}$ should at least be the order of magnitude of the largest static energy among the quarks', $174.9 \mathrm{GeV}$ $(174.3 \pm 5.1 \mathrm{GeV})$, the top quark's static energy. Let $J_{2}=179.4 \mathrm{GeV}$, and the $\langle D\rangle$ instead of the $D_{\min }$, we obtain by Equation (25)

$$
T_{C} \approx 1.6 \times 10^{17}{ }^{\circ} \mathrm{K}
$$


The value of the $T_{C}$ calculated by the $J_{1}$ is too small to be adopted.

\subsection{The Temperature Region of Cosmic Inflation}

By the reference [3], we know that the dark particles are also involved in the superlight particles. In the temperature region $T_{E W}<T \leq T_{C}$ all particles travel at the superlight speed in the fractal structures. The more closely to $T_{E W}$ the temperature reaches, the bigger the sizes of the sub-blocks and the blocks turn into, the higher the fractal dimensions will be, and the faster than the light speed the particles' velocities will become (see Equation (23)), and the more room will be full of the particles. The particles' behavior results directly in the cosmic inflation [8] [9], the huger inflation will take place in the vicinity of the $T_{E W}$. The maximum fractal dimension $D_{\max }=3$ is given by the Equation (5) of the reference [5] when the block's side $n$ tends to infinity, $n \rightarrow+\infty$. We then get the fastest limiting speed $V_{B, \max }$ of the inflation through Equation (23),

$$
V_{B, \max }=(\sqrt{3} / 3) D_{\max }^{2} c>5.1 c
$$

More than five times the light speed! We see that the superlight phenomena exist on all hierarchies, so the inflation is uniform in the sense of both locality and globality.

\subsection{Dark Energies}

Since the fractal structures exist in the thermal equilibrium state, the cosmic energies distribute evenly in the three spacetimes. The sub-blocks and the blocks lie on the same hierarchy and share the same 4-dimansional spacetime occupied by one third of the energies (about 33.3\%), the relevant particles get masses under the Higgs field when the temperature decreases, $T \leq T_{E W}$, and the energies are measurable. The $(D+1)$-dimensional spacetime and the $\left(D^{4}+1\right)$-dimensional spacetime are full of two thirds of the energies (about 66.7\%), corresponding to the accelerating expansion of the universe, they are dark energies [10]. Because the superlight particles keep their original high speeds after the electro-weak phase transition and the particles of the 4-dimensional spacetime, which speeds are no more than the light velocity, catch up impossibly with them, therefore, the energies cannot be measured.

\subsection{Effect of Higgs Field on Weak Interaction and Spins' Chirality}

The first effect is that the Higgs particles can transfer into $W^{+}, W^{-}$, and $Z$ particles. The second, the field as a background maintains the breaking of the time local inverse symmetry. According to the statistical mechanism the uniform space embodies that the trajectory of a representative point passes, in the time course, through any neighborhood of any point of the relevant region of the phase space. The mean value $\langle f\rangle$ of a physical parameter $f(t)$ is defined as

$$
\langle f\rangle=\lim _{T \rightarrow+\infty} \frac{1}{T} \int_{-T / 2}^{T / 2} f(t) \mathrm{d} t
$$

for the time inverse symmetry, corresponding to the detailed balance principle: a 
tendency of a physical system to transfer one state to another must be counterbalanced by an identical tendency to switch between the tendency states in the reverse direction. The duality between the time and the energy is an intrinsic property of the nature [11], not only for their values, but also for their polarity. An antiparticle posses the energy reversing to the particle's, corresponding to the time inverse transformation. This idea is formulated as a rule of the Feynman diagrams: for instance, for two incident particles, electron and positron, the time direction referring to the latter is drawn by a reverse arrow against the electron's. For the weak interaction system the $f(t)$ is assigned as the particle's spin or its energy. As the spin, $f(t)>0$ means the spin-up state; and $f(t)<0$, the spin-down state. The $f(t)<0$ can be rewritten as $f(-t)=-f(t)<0$, corresponding to the time inverse, which means if the time is inverse symmetry, so $\langle f\rangle=0$, the two spin states have the same probability. As the energy, $f(t)>0$ refers to the particle's energy, $f(-t)=-f(t)<0$ to its antiparticle's. If $\langle f\rangle=0$, the two types have the same total number since the time inverse symmetry. When Higgs field is established not all of lattices have the positive time direction unless at the absolute zero. The breaking of the time inverse symmetry holds locally, Equation (28) should be revised by

$$
\langle f\rangle=\lim _{t \rightarrow+\infty} \frac{1}{T} \int_{-(T / 2-\Delta)}^{T / 2+\Delta} f(t) \mathrm{d} t
$$

where $0<\Delta<T / 2$. For weak interaction the force range is too small to have an infinite equilibrium period. The real equilibrium period should be finite, denoted as $T^{\prime}, 0<2 \Delta^{\prime}<T^{\prime} \ll T$, and $N T^{\prime}=T$. Therefore, Equation (29) changes into

$$
\begin{aligned}
\langle f\rangle & =\lim _{N \rightarrow+\infty} \frac{N}{N T^{\prime}} \int_{-\left(T^{\prime} / 2-\Delta^{\prime}\right)}^{T^{\prime} / 2+\Delta^{\prime}} f(t) \mathrm{d} t \\
& =\frac{1}{T^{\prime}} \int_{-\left(T^{\prime} / 2-\Delta^{\prime}\right)}^{T^{\prime} / 2-\Delta^{\prime}} f(t) \mathrm{d} t+\frac{1}{T^{\prime}} \int_{T^{\prime} / 2-\Delta^{\prime}}^{T^{\prime} / 2+\Delta^{\prime}} f(t) \mathrm{d} t \\
& =\frac{1}{T^{\prime}} \int_{T^{\prime} / 2-\Delta^{\prime}}^{T^{\prime} / 2+\Delta^{\prime}} f(t) \mathrm{d} t>0
\end{aligned}
$$

where the integral region is $0<T^{\prime} / 2-\Delta^{\prime} \leq t \leq T^{\prime} / 2+\Delta^{\prime}<+\infty$. Equation (30) states that the symmetries of the spins and the particles' energies are statistically broken [12] [13]. The space lattice model indicates the particles such as S bosons are independent of the time [3], Equation (30) is not available to them, which may be the primary reason why these particles are just their antiparticles.

The space field has both right-handed system and left-handed system, each system includes its own gradient field and curl field, shown as Equations (8), (9), (10), and (11) of the reference [3]. According our theory [14], electric charge stimulates the right-handed system, requiring sufficiently and necessarily the right-handed spins, and mass only another system the left-handed spins are applicable to, vice versa. Therefore, electrons' spins are chiral symmetric, and neutrinos' spins are only left-handed rotation [15]. Instead of $t$, the negative time $-t$ makes the left-handed system change into the right-handed system, relating to the antineutrinos' spins. Since the $S$ bosons are the quanta of the space field, 
their spins are of chiral symmetry, and the photons and the gravitons as the excitation states of the $\mathrm{S}$ bosons maintain the characteristics [3].

\section{Conclusion}

In the evolution of the universe the time underwent three phase transitions with three characteristic temperatures. The fractal structures of the spacetime possess super symmetries and hierarchies under the gauge invariance, in which we have found the cosmic inflation and dark energies. The concepts of the mass charge and the Higgs potential make us understand the meaning of the mass. Further, the concept of mass tensor is suggested if the time warps. The particles' helicities are determined by the space field.

\section{References}

[1] Mandelbrot, B.B. (1983) The Fractal Geometry of Nature. W.H. Freeman and Company, New York.

[2] Feng, Y. (2016) Journal of Modern Physics, 7, 536-542. https://doi.org/10.4236/jmp.2016.76056

[3] Feng, Y. (2016) Journal of Modern Physics, 7, 1483-1491. https://doi.org/10.4236/jmp.2016.712134

[4] Goldstone, J., Salam, A. and Weinberg, S. (1962) Physical Review, 127, 965-970. https://doi.org/10.1103/PhysRev.127.965

[5] Feng, Y. (2014) American Journal of Modern Physics, 3, 184-194. https://doi.org/10.11648/j.ajmp.20140304.16

[6] Weinberg, S. (1993) The First Three Minutes. Perseus Books Group, New York.

[7] Kirzhnits, D.A. and Linde, A.D. (1972) Physics Letters B, 42, 471-474. https://doi.org/10.1016/0370-2693(72)90109-8

[8] Guth, A., Kaiser, D. and Nomura, Y. (2014) Physical Letters B, 733, 112-119. https://doi.org/10.1016/j.physletb.2014.03.020

[9] Guth, A. (1981) Physical Review D, 23, 347-356. https://doi.org/10.1103/PhysRevD.23.347

[10] Peebles, P.J.E. and Bharat, R. (2003) Reviews of Modern Physics, 75, 559-606. https://doi.org/10.1103/RevModPhys.75.559

[11] Feng, Y.G. (2014) Natural Science, 6, 1149-1158. https://doi.org/10.4236/ns.2014.614103

[12] Wu, C.S., Ambler, E., Hayward, R.W., Hoppes, D.D. and Hudson, R.P. (1957) Physical Review, 105, 1413-1415. https://doi.org/10.1103/PhysRev.105.1413

[13] Christenson, J.H., Cronin, J.W., Fitch, V.L. and Turlay, R. (1964) Physical Review Letters, 13, 138-140. https://doi.org/10.1103/PhysRevLett.13.138

[14] Feng, Y. (2015) Journal of Modern Physics, 6, 573-577. https://doi.org/10.4236/jmp.2015.65062

[15] Goldhaber, M., Grodgins, L. and Sunyar, A.W. (1958) Physical Review, 109, 1015 1017. https://doi.org/10.1103/PhysRev.109.1015 
Submit or recommend next manuscript to SCIRP and we will provide best service for you:

Accepting pre-submission inquiries through Email, Facebook, LinkedIn, Twitter, etc. A wide selection of journals (inclusive of 9 subjects, more than 200 journals)

Providing 24-hour high-quality service

User-friendly online submission system

Fair and swift peer-review system

Efficient typesetting and proofreading procedure

Display of the result of downloads and visits, as well as the number of cited articles Maximum dissemination of your research work

Submit your manuscript at: http://papersubmission.scirp.org/

Or contact jmp@scirp.org 